\title{
Power Saving Strategies in Green Cloud Computing Systems
}

\author{
Amlan Deep Borah ${ }^{1}$, Deboraj Muchahary ${ }^{2}$, Sandeep Kumar Singh ${ }^{3}$ and Janmoni \\ Borah $^{4}$ \\ 1, 2, 3, 4 National Institute of Technology, Arunachal Pradesh - 791112, India \\ 1amlan20deep@gmail.com, ${ }^{2}$ debomuchahary@yahoo.com, \\ ${ }^{3}$ sandeeps177@gmail.com, ${ }^{4}$ borah1989@gmail.com
}

\begin{abstract}
The most challenging field of concern these days is energy conservation in various applications. Energy crisis led a way to green computing and green computing needs an efficient redesign of algorithms and mechanisms to meet the desired energy efficiency. Green IT is a study and practice which can reduce energy consumption significantly. In this paper various efficient energy saving Green IT methods are discussed. As cloud computing has become a fast emerging technology this days, some energy saving strategy for cloud computing has also been discussed in the paper. Most of the IT sector energy consumption happens in data centers. Virtual Machine migration an emerging energy aware technology used in data centers is also discussed in the paper. Finally some power conserving matrix parameters are given importance.
\end{abstract}

Keywords: VM migration, Green IT, Cloud Computing, DVFS, Energy Saving

\section{Introduction}

Energy saving has become a primary issue because of economic, environmental and marketing aspects of energy in every field. This concern has a strong influence on the information and communication technology sector, electronics designers and more specifically the networking field etc. Networking infrastructure and Datacenters involves high-performance and high-availability machines. Therefore they contain high energy-consuming powerful devices which require air conditioning to sustain their normal operation. Research says Information and Communication Technology (ICT) industry constitutes $2 \%$ of global total CO2 emissions and $3 \%$ of global energy expenditure from the energy and environmental point of view [1]. Data centers consume maximum energy in ICT [2]. The network infrastructure of a data centre consumes nearly $30 \%$ of the total power consumption used for computation which is basically composed of links and switches. The energy consumption of a link depends on its capacity rather than directly its utilization. Total power consumption by data centre in 2012 was around 38 Giga Watt (GW) [3] which can fulfils the energy requirements of all residential households of United Kingdom if it would have been utilized properly. This is around $63 \%$ more than the power consumption of 2011 [4]. The main sources of power Consumption in a data centre are cooling, computing resources, and network elements, where cooling constitutes $40 \%$ of the total energy consumption in a data centre. As Cloud computing becomes more scattered, these increasing data storage and computation needs raise the energy consumption of their large infrastructures [5]. That's why energy consumption has become a main factor in designing modern Cloud environment. Greening...”Our Common Future", a report issued by the World Commission on Environment and Development in 1987 proposed the basic concepts of "sustainable development". Immediately this idea was recognized by general environmentalists, social activists, economists and the international community [6], 
[7]. The U.S. Environmental Protection Agency (EPA) [8], in 1992 launched consumer Energy Star plan, which was designed to reduce energy consumption and decrease Greenhouse gas emissions caused by power usage

Basically Green Computing can be studied in two aspects: software technology and hardware technology $[9,10,11]$. Borah et al., have describes various power saving strategies for greening cellular base stations [12]. Software technology includes lots of aspects, design methods to enhance program efficiency, reduce storage space and save energy, offers computing modes such as High Performance Computing, Distributed Computing and Cloud Computing etc. In the hardware side technologies are adopted to reduce energy consumption, $\mathrm{CO} 2$ emissions, and can increase economic efficiency and recycling technology. At the same time, Humanbeing engineers can afford technical support to the cause. Thus greening cloud computing become a key issue in field of computer base journey.

Li et al., [13] has proposed an optimized cost model for calculating total cloud utilization cost. Innovative interconnect technology is one another such prime approach for $e$. g., three dimensional stacking technology [14]. It uses CMP (chip multiprocessor architecture) which greatly reduces power consumption. Pico Server [15] is one such architecture that utilizes 3D technology to join several simple slow processor cores with multiple DRAM dies that form the primary memory. Power efficient data transfer between processor to processor or processor to memory or processor to input-output (I/O) have been given in [16] for diminishing power requirements of a server.

The comparison of adaptive link rate and sleep mode for wired networks is studied in [17] where the authors explained the design and evaluation of forms of power management schemes that reduce the networks energy consumption . The first is by taking ideal network components to sleep mode and secondly adjust the network operation rate based on offered workload. This result show 50\% energy saving for lightly utilized networks $(10-20 \%)$. Heller et al., in [18] proposed a dynamic power management scheme named Elastic Tree for data centre network. It determines the set of active network elements such as switches and links dynamically. For testing author implemented Elastic Tree on Open Flow [19] switches. The results showed up to 50\% of network energy. In [20] Mahadevan et $a l$, proposed an energy aware algorithm for data centre networks, where author consider sleep modes for components of network devices rather than completely putting the network device into sleep mode.

Fisher et al., in his research proposed turning off individual cables and Network Interface Cards (NICs) rather than links [21], because the links in core networks are "bundles" of multiple physical cables and line cards. Here authors proposed three heuristics Fast Greedy Heuristic, Exhaustive Greedy Heuristic, and Bi-level Greedy Heuristic to solve the problems occurred. The energy savings of the heuristics are very close to each other but differ in running time. Using Abilene traffic data, authors saved $79 \%$ energy for bundles of five cables in each link.

A cross study in [22] by Kliazovich et al., proposed DENS a network-awaked methodology while making energy efficient scheduling in data centres. DENS method tries to minimize the energy consumption by scheduling jobs to computing resources according to best fit algorithm, while taking into account load level and the communication potential of the network components. Its main objective is to avoid congestion while minimizing the number of computing servers to meet the strict QoS requirements of jobs while slightly increasing the energy consumption.

In 2006, the American National Standards Institute (ANSI), under the operation of the United States Green Electronics Council, formally adopted Standard No. 1680 of the American Association of Electrical and Electronic Engineers (IEEE) [9], and 
implemented EPEAT certification system, which was used to certify electronic products by checking its environmental impact during their life cycle.

\section{Some Energy Conserving Strategies}

\subsection{Use Renewable Energy Sources}

All datacenters of clouds maximum time use generator to provide backup power and with data center $\mathrm{CO} 2$ dissipation and also GHGs. Instead of it renewable energy sources such as hydro energy, wind energy, solar energy should be sued to generate electricity for fulfilling the power requirement and cooling requirement of data center to save energy and environment form pollution. Because only Google data centers around the world continuously draw almost 260 million watts which is about a quarter of the output of a nuclear power plant.

\subsection{Nano Data Centers}

Increased concern over energy consumption in modern typical data centers, a new, distributed computing platform called Nano Data Centres $(\mathrm{NaDa})$ is preferred. $\mathrm{NaDa}$ provides computing and storage services and adopts a managed peer-to-peer model to form a distributed data centre infrastructure. Instead of few large data centres it consists of large number of geographically distributed nano data centres which are of smaller in sized, interconnected and spread along the network edges. In energy consumption VoD access model data access from $\mathrm{NaDa}$ saves at least $20 \%$ to $30 \%$ of the energy compared to traditional data centres [23]. Energy consumed in various parts of data centre is shown in Figure 1.

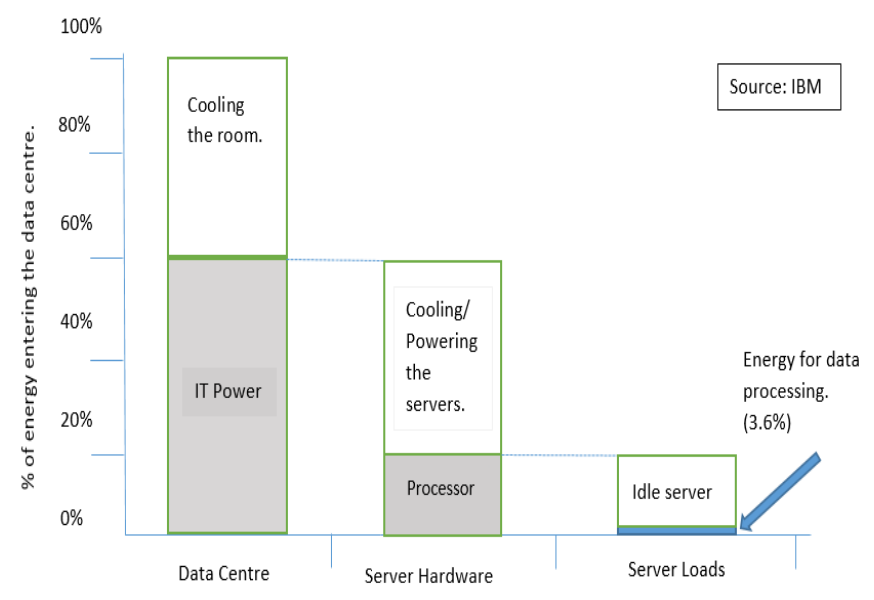

Figure 1. Energy Consumed in Various Parts of Datacenter

\subsection{By using Energy Efficient Storage}

Storage of cloud should be replaced by energy efficient storages components. As the life time of a data centre is limited up to 9 to 10 years, so while renovating existing data centre developer should has to use energy efficient memory for e.g., solid state storage and other updated efficient storage devices. Solid state storage has no moving mechanical component as hard disk drive because of which it requires less cooling as compared to hard disk drive, causes less energy requirement for cooling. 


\subsection{By reducing CPU Power Dissipation}

Processor consumes electrical energy in form of charging for its operation, for its switching devices and for cooling of transistors and numerous chips. It also dissipates this energy in surroundings in the form of heat. By adapting free cooling this power dissipation can be reduced.

\subsection{By using Advance Clock Gating}

Clock gater is a hardware switch to activate and deactivate the clock pulse. The clock of a logic block must be activated only when the logic block is doing some work and must be turned off when logic block is in ideal mode. This popular technique has been used in many synchronous circuits but it can also be used in globally asynchronous and locally synchronous circuits [24] for reducing dynamic power dissipation.

\subsection{By Reducing Cooling Requirements}

Previously cooling was done by mechanical refrigerator that takes service of compressor inside the data centre or externally chilled water is supplied to main air handler for the cooling its IT equipment. Now free cooling system is developed to optimize the power requirement of cooling instead of mechanical cooling. This newly adopted technique says if the air temperature of outside world is below or at the critical point, then the mechanical refrigerator can provide direct or indirect cooling by itself. It does not reduce the required fan energy for cooling it only eliminates the need of mechanical cooling energy.

\subsection{By Saving Strategy of Hardware Temperature Control}

Hardware temperature control energy saving strategy includes two aspects: one is to computer hardware (such as servers, storage medium, etc.) and cooling measures, another is temperature control mechanism for processor, storage hardware and its temperature shock. Some scholars have carried out extensive research in this regard, such as the blade server system, Solid State Disk (SSD) storage space, IBM's "electron spin" storage technology, APC "thermal channel sealing system" and so on. When upper application of load and operation characteristics changed, the processor and other hardware may be caused temperature raise. Therefore, energy consumption rising problem is produced. It became necessary thermal sensor technology around the hardware for real-time monitoring and dynamic intelligent cooling to hardware based on the monitoring results.

On the other hand, large ICT companies like Google displaced their server farms to the banks of the Columbia River to take advantage of the energy offered by the nearby hydroelectric power plants. The river water flow may in addition be used within the cooling systems, as experimented by Google [25] and it also take the advantage of cold environment of the river bank in the cooling prospective. In Tent and Marlow projects [26] an alternative cooling system is investigated by Microsoft by leaving servers in the open air so that heating dissipates more easily [27].

\subsection{Server Consolidation}

In most of the time one physical server uses only less than $10 \%$ of total CPU utilization. So it is better to consolidate several roles in a single physical server i.e. migrating server roles from different underutilized physical servers onto virtual machines. This reduces total number of hardware and energy consumption. 


\subsection{By using Energy Saving for Computer Architecture}

Cloud computing is consist of a set of large number of computers. Heterogeneous computer is consisting of multiple processors which are relatively independent processors of different type. In such systems using the generally used processors as the main control unit and other special purpose processors as the accelerating unit gives an efficient energy consumption ratio. Fast Array and Wimpy Nodes (FAWN) is a cluster system with low power consumption capability proposed by David Andersen, Carnegei Mellon University, for large scale data intensive applications [28].

\subsection{Energy Saving Strategy for Compiling Technology}

Optimization of compiler technology also takes a major role in energy saving activity. A better compilation technology optimizes the application technology as well as helps analysing the behaviours of application programs to minimize the system or processor operation power consumption. A model is proposed by Zhao Rongcai in [29] which is used to reduce the execution frequency to reduce the power consumption by multithreading system structure.

\subsection{Energy Saving Strategy of Application Software Power}

With the application of some method to the source program structure level it is possible to optimize software power. For example cycle structure optimization techniques have been reaslized in the compiler and it gives a good power optimization result [29]. Application software power consumption strategy focuses on the reduction of space complexity and time complexity of the algorithm to reduce the system power. This can be achieved by implementing some effective algorithms viz. compression data storage space etc. which reduces repeated calculation and redundancy of the algorithm.

\subsection{Energy Saving Strategy of System Software}

This strategy includes energy efficient scheduling between nuclear, equipment resource management and dynamic energy consumption management of operating system. The former represents an energy efficient scheduling method in poly nuclear system. Wang Jing analyzed thread scheduling strategy and resource classification mechanism for reducing resource contention and discussed the possible future research direction of development [30]. The equipment resource management maintains an optimal environment for all the individual computing elements. Dynamic energy consumption refers to the operating system dynamically manipulates the system unit to obtain minimum power consumption without degrading the task assigned to be performed.

\subsection{Energy Saving Strategy of Virtual Machine Manager}

This strategy draws in it these aspects: Energy management in interface support, in framework and desk-top class virtual machine and many more. Stoess in [31] proposed a framework for energy management in virtualized server. Some major area of research in cloud computing to manage energy consumption are model of energy management and task scheduling strategy among operating systems, virtual machine manager and upper application of hardware.

\subsection{Live Migration of Virtual Machine (VM)}

Live migration is nothing but moving a running VM from one host to another. It provides various benefits to data centers such as load balancing, power management 
and transparent IT maintenance. The cost of this kind of live migration is affected by some parameters [32]. Parameters related to physical machine is resource utilization of source and host systems. The available average network bandwidth between source and target host is also a parameter in the migration of VM. In addition to live migration of VM green cloud architecture switches off the underutilized servers to meet minimum power consumption without losing quality of service.

\subsection{Energy Saving Strategy of Network Environment}

Cloud computing is a networked platform. So, by applying some energy management strategy a quite good result can be obtained. Energy management in network protocols and algorithm is the focal point of this strategy. A large number of network protocols and algorithms do not meet the requirement of energy saving. Some such protocols are viz. TCP, CSMA/CD, and CSMA/CA in wireless network. Moreover, in the literature works on adaptive link rate and sleep modes. The adaptive link rate methods dynamically adjust the data rate of the links according to the traffic requirements. Another way sleep mode technique achieves energy saving by turning off the component or switch to sleep mode of a subset of idle components. At the same time the remaining active elements should still satisfy the fluctuating requirements. However the QoS requirements should be satisfied and fault tolerance should be completely avoided.

\subsection{Task Consolidation for Efficient Energy Consumption}

The task consolidation means assigning a set of $\mathrm{n}$ tasks to a set $\mathrm{r}$ of cloud resources without violating the time constraint to minimize energy consumption. The author in [33] described that the energy consumed by a task can be calculated from the information of its processing time and processor utilization. For a given resource $r_{i}$ at any given time the utilization is defined as:

$$
U_{i}=\sum_{j=1}^{n} u_{i, j}
$$

Where, $\mathrm{n}=$ number of task running at that time and $\mathrm{u}_{\mathrm{ij}}=$ resources used by a task.

The assignment of task to a resource must be very carefully handled. Because a task which requires $70 \%$ resource utilization cannot be assigned to a resource which is associated with only $50 \%$ resource utilization at the time of the task arrival. The energy saving can also be met using slack reclamation with the support of dynamic voltage/frequency scaling (DVFS). The DVFS technique enables energy consumption by lowering the voltage and sacrificing the speed of the system. Some energy efficient task consolidation algorithms are Energy Consolidation and Task Consolidation (ECTC) and Maximum Utilization (MaxUtil).

\section{Conclusion}

We have analyzed various energy saving strategies in data centers and cloud computing environment. Various energy effective strategies should be implemented in data centers to make an eco-friendly data centers. Cloud computing is effective only if energy consumed in servers are saved. Task consolidation especially in cloud computing has become an important approaches to streamline resource use and improve energy efficiency. Energy saving strategies saves a sufficient amount of power and of course cut down the cloud footprint. 


\section{Acknowledgements}

We would like to thank Mr. Anish Kr. Saha and Mr. Koj Sambyo (Asst. professor, Dept of CSE, NIT Arunachal Pradesh) for their motivation, guidance and support throughout the work who instead of their busy schedules, always guided us in the right direction.

\section{References}

[1] "Smart 2020: Enabling the low carbon economy in the information age," The Climate Group SMART 2020 Report, Tech. Rep., June 2008.

[2] U. E. P. Agency, "Data centre report to congress," U.S. Environmental Protection Agency, Tech. Rep., 2007.

[3] "Taxes and incentives for renewable energy", KPMG international, June 2012.

[4] Anubha Jain, Manoj Mishra, Sateesh Kumar Peddoju and Nitin Jain” Energy Efficient ComputingGreen Cloud Computing” in IEEE 2013.

[5] Arthi $\mathrm{T}$ and Shahul Hamead H, "ENERGY AWARE CLOUD SERVICE PROVISIONING APPROACH FOR GREEN COMPUTING ENVIRONMENT “in IEEE 2013.

[6] Guo Bing, Shen Yan, and Shao ZL, "The Redefinition and Some Discussion of Green Computing", Chinese Journal of Computers, vol. 32, Dec. 2009, pp. 2311-2319.

[7] Zhang Juntao, "Urban and regional sustainable development", Dongbei University of Finance and Economics Press, 2008.9.ISBN $\square$ 9787811224573. (In Chinese)

[8] US Environmental Protection Agency. http://www.epa.gov.

[9] IEEE Standard 1680-IEEE Standard for Environmental Assessment of Personal Computer Products, Including Laptop Personal Computers, Desktop Personal Computers, and Personal Computer Monitors. Standards Activities Board of the IEEE Computer Society.2006.

[10] Mydhili K Nair, Dr.V.Gopalakrishna, "Generic Web Services: A Step Towards Green Computing", International Journal on Computer Science and Engineering, Vol.1, Mar. 2009, pp. 248-253.

[11] Sergi Figuerola, Mathieu Lemay, Victor Reijs, Michel Savoie, Bill St.Arnaud, "Converged Optical Network Infrastructures in Support of Future Internet and Grid Services using IaaS to Reduce GHG Emissions", Journal of Lightwave Technology, Vol. 27, Dec. 2009, pp. 1941-1946.

[12] J. Borah, S. K. Singh, A. D. Borah, "Cellular Base Station and its Greening Issues", International Journal of Advanced Electronics and Communication Systems (CSIR-NISCAIR Approved), Vol.3, No.2, pp.1-4, April-May 2014.

[13] L. Liu, H. Wang, X. Liu, X. Jin, W. He, Q. Wang, and Y. Chen "Green Cloud: a new architecture for green data centre," in IEEE Conference on Autonomic Computing (ICAC), Barcelona, Spain, 2009, pp. 29-38.

[14] Taeho Kgil, David Roberts and Trevor Mudge "PicoServer: Using 3D Stacking Technology to Build Energy Efficient Servers”, ASPLOS'06October 21-25, 2006, San Jose, California, USA.

[15] www.techarp.com.

[16] J. Chabarek, J. Sommers, P. Barford, C. Estan, D. Tsiang, and S. Wright, "Power Awareness in Network Design and Routing," in The 27th Conference on Computer Communications IEEE INFOCOM 2008, 2008,pp. $457-465$.

[17] S. Nedevschi, L. Popa, G. Iannaccone, S. Ratnasamy, and D. Wetherall, "Reducing network energy consumption via sleeping and rate-adaptation," in Proceedings of the 5th USENIX Symposium on Networked Systems Design and Implementation, Berkeley, CA, USA, 2008, pp. 323-336.

[18] B. Heller, S. Seetharaman, P. Mahadevan, Y. Yiakoumis, P. Sharma, S. Banerjee, and N. McKeown, "Elastictree: saving energy in data centre Networks," in Proceedings of the 7th USENIX conference on Networked Systems design and implementation, 2010.

[19] "The Open Flow Switch.” [Online]. Available: http://www. openflowswitch.org

[20] P. Mahadevan, P. Sharma, S. Banerjee, and P. Ranganathan, "Energy Aware Network Operations," IEEE INFOCOM Workshops 2009, pp. 1-6, Apr. 2009.

[21] W. Fisher, M. Suchara, and J. Rexford, "Greening backbone networks: reducing energy consumption by shutting off cables in bundled links," in Green Networking, 2010, pp. 29-34.

[22] D. Kliazovich, P. Bouvry, and S. Khan, "DENS: Data Centre Energy- Efficient Network-Aware Scheduling," in Green Computing and Communications (GreenCom), 2010, pp. $69-75$.

[23] Derya C, avdar and Fatih Alagoz, "Survey of Research on Greening Data Centres" in Symposium on Selected Areas in Communications Globecom 2012.

[24] Jonas Carlsson, Kent Palmkvist, and Lars Wanhammar, "A Clock Gating Circuit for Globally Asynchronous Locally Synchronous Systems", Norchip Conference, 2006.

[25] R. H. Katz, "Tech Titans Building Boom,” IEEE Spectrum, vol. 46, pp. 40-54, Feb. 2009.

[26] R. Barga, "Cloud Computing - A Microsoft Research Perspective.” Keynote Speech at IEEE P2P 2009 (Seattle, WA, USA), Sept. 2009. 
[27] Aruna Prem Bianzino, Claude Chaudet, Dario Rossi, and Jean-Louis Rougier, “A Survey of Green Networking Research" in IEEE COMMUNICATIONS SURVEYS \& TUTORIALS, VOL. 14, NO. 1, FIRST QUARTER 2012.

[28] D.G., Andersen, et al."FAWN: A fast array of wimpy nodes". 2009.

[29] Zhao, R. et al," A Multithreaded Compiler Optimization Technology with Low Power". Journal of Software, 2002 pp. 1123-1129.

[30] J., Wang et al.” A Survey of CMT Thread Schedule Policies”. Computer Science 2007(9), pp. 256$258+289$.

[31] Stoess, C. Lang, J. and F. Bellosa. "Energy Management for Hypervisor based Virtual Machines". 2007.

[32] "Costs of Virtual Machine Live Migration" A Survey Anja Strunk Chair of Computer Networks Faculty of Computer Science Technical University of Dresden 01062 Dresden, Germany.

[33] R. Yamini."Power Management In Cloud Computing Using Green Algorithm" IEEE-International Conference On Advances In Engineering, Science And Management (ICAESM -2012) March 30, 31, 2012. pp. 128-133.

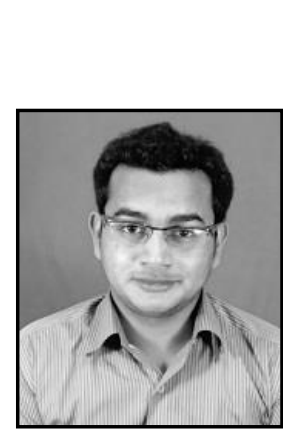

\begin{abstract}
Authors
Amlan Deep Borah, received his B.Tech degree in Electronics and Communication Engineering from Central Institute of Technology, Kokrajhar, India in 2013. He is currently pursuing M.Tech in Mobile Communication and Computing at National Institute of Technology, Arunachal Pradesh, India. His research interest includes Digital signal processing, VLSI design, Cloud and Green computing. He has published some research papers in International journals and conferences.
\end{abstract}

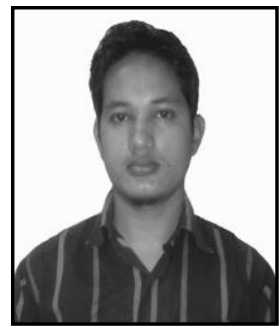

Deboraj Muchahary, received his B.Tech degree in Electronics and Communication Engineering from Central Institute of Technology, Kokrajhar, India in 2013. He is currently pursuing M.Tech in Mobile Communication and Computing at National Institute of Technology, Arunachal Pradesh, India. His research interest includes Digital signal processing, VLSI design and Green computing. He has published some research papers in International journals and conferences.

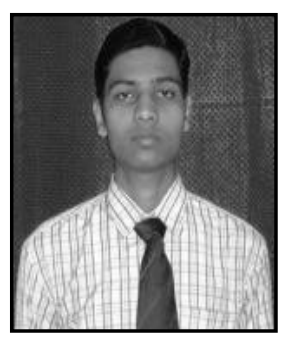

Sandeep Kumar Singh, received his B.Tech degree in Electronics and Communication Engineering from National Institute of Technology, Agartala, India in 2012. He is currently pursuing M.Tech in Mobile Communication and Computing at National Institute of Technology, Arunachal Pradesh, India. His research interest includes Network on chip, VLSI design and Green-cloud computing. He has published some research papers in International journals and conferences.

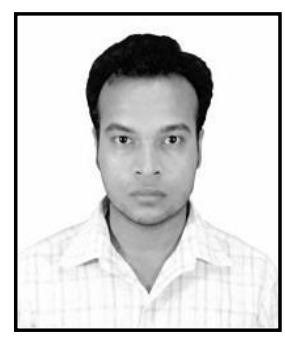

Janmoni Borah, received his B.Tech degree in Electronics and Communication Engineering from Central Institute of Technology, Kokrajhar, India in 2013. He is currently pursuing M.Tech in Mobile Communication and Computing at National Institute of Technology, Arunachal Pradesh, India. His research interest includes Mobile communication, Microstrip antenna design for multi-band applications, Microstrip filter design and Green computing. He has published some research papers in International journals and conferences. 